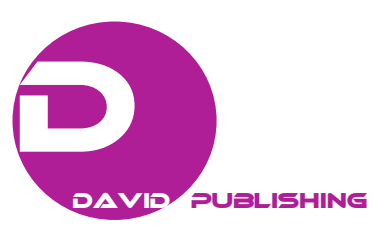

\title{
Design Integrated Distillation Columns for Binary
}

\section{Systems}

\author{
Jesús Enrique Ceballos Jiménez ${ }^{1 *}$ and Carlos Eduardo Segura Garcia ${ }^{2}$ \\ 1. Petrochemical Research and Development Unit, Petrochemical Venezuela SA (Pequiven), Valencia 2003, Venezuela \\ 2. Faculty of Engineering, University of Carabobo, Naguanagua 2005, Venezuela
}

\begin{abstract}
The purpose of this study is to contribute methodologically, through a systematic study of the application of different optimization techniques to design integrated distillation columns in binary, for it established one optimization technique, deterministic, was optimized distillation column at steady state. For this part was used tabu search to solve the type binary problem. For each choice of the search whole continuous variables were optimized with the deterministic method for process design perturbation is applied to the molar fraction of the feed. It develops and implements the mathematical algorithm solving the problem, then validation of the methodology proposed dynamic simulation of the system is compared with and without control, with both traditional and integrated approaches.
\end{abstract}

Key words: Integrated design, deterministic, optimization.

\section{Introduction}

In recent years, several researchers have pointed out the need to consider the characteristics of controllability in the initial stages of design to alert engineers to potential problems and provide some means of selection within the alternatives.

The interaction between these two disciplines arises because the chemical process design of an inherently determines its controllability, which qualitatively means how well a process is able to reject disturbances, how severely the multiple variables interact and how easily the system moves from one operating point to another.

There is an optimum for the process design procedure, called integrated design process, which is to integrate in the design task process restrictions with system controllability [1].

The integrated design is the technique of design processes by which physical plant parameters that minimize operating costs and construction, while

*Corresponding author: Jesús Enrique Ceballos Jiménez, M.Sc., research filed: process engineering. E-mail: ceballos.jesus@gmail.com. complying with the characteristics of controllability imposed on the process are obtained [2].

This type of approach would automatically select, within the same algorithm solving the problem of design, the optimal structure of the plant with the controller structure, obtaining a plant whose units are connected in an optimal way, its sizing represents the minimum construction cost could satisfy the requirements for proper operation of the process. Simultaneously optimal controller parameters that provide a dynamic system behavior set at design stage would be obtained.

For all this, it is crucial to the existence, development and documentation of works like the one below is presented, allowing methodological strategies to clear the way for designers and process control.

Throughout the study, has made a significant effort to explore optimization techniques for integrated design of binary distillation column.

All this effort is aimed to contribute methodologically to providing the best options to process designer, providing a detailed breakdown of the methodology for the simultaneous optimization of design and control through a mathematical algorithm 
solution for the case study.

\section{Experimental Sections}

\subsection{The Integrated Design Approach}

Gutiérrez and Prada [3] note that the "integrated design" is a technique or approach to plant design whereby the characteristics of controllability are previously considered in the design stages, allowing to set dynamic system specifications that facilitate the and flexibility of operation that can be the same governed by an automatic control.

The idea of integrated design is based on the possibility of designing processes optimization criteria, incorporating conditions that guarantee a good dynamic performance of the system in all the early stages of design. In this way both the plant parameters of the controller are obtained simultaneously.

\subsection{System under Study}

This proposed approach to the design of a binary distillation column, the goal is to design a process that separates a liquid feed funds saturated products and distillates. The number of plates, feed location, column diameter, flow rates and compositions are calculated.

To develop the mathematical model constant relative volatility $(\alpha)$, a partial reboiler and a total condenser is assumed. The alternative structure is represented by binary variables $p_{i}$ and $q_{i}$, which represent the residence of the feed and reflux respectively for each plate $i$.

The invariant continuous time variables in the model are: accumulation of fluid in the plate, $\mathrm{M}$, hydraulic time constant on the plate, $\beta$, reflux ratio at steady state, Rss, steam in the boiler at steady state, Vss, diameter the column, Dc, controller gains and time constants Kv, KR, TV, TR.

The dynamic variables in the model are liquid compositions, $x_{i}$, vapor compositions, $y_{i}$, liquid flow rate for each dish, $L_{i}$, underflow and distilled, $B$ and $D$, steam boiler, $V$, and reflux, $R$.
The proposed design must be optimal gain both economic criteria and the controllability of the process ensuring a feasible operation [4].

Fig. 1 shows the diagram of the system under study.

\subsection{Optimization Techniques}

Classical methods: Methods or deterministic local search are studied, specifically the optimization technique based on gradient search.

Heuristics and Stochastic methods: Genetic Algorithms, being itself a global optimization method, the probabilistic method is used based on population.

The primary objective pursued by the design of the process under study is to determine the optimal physical dimensions (diameter, number of trays and

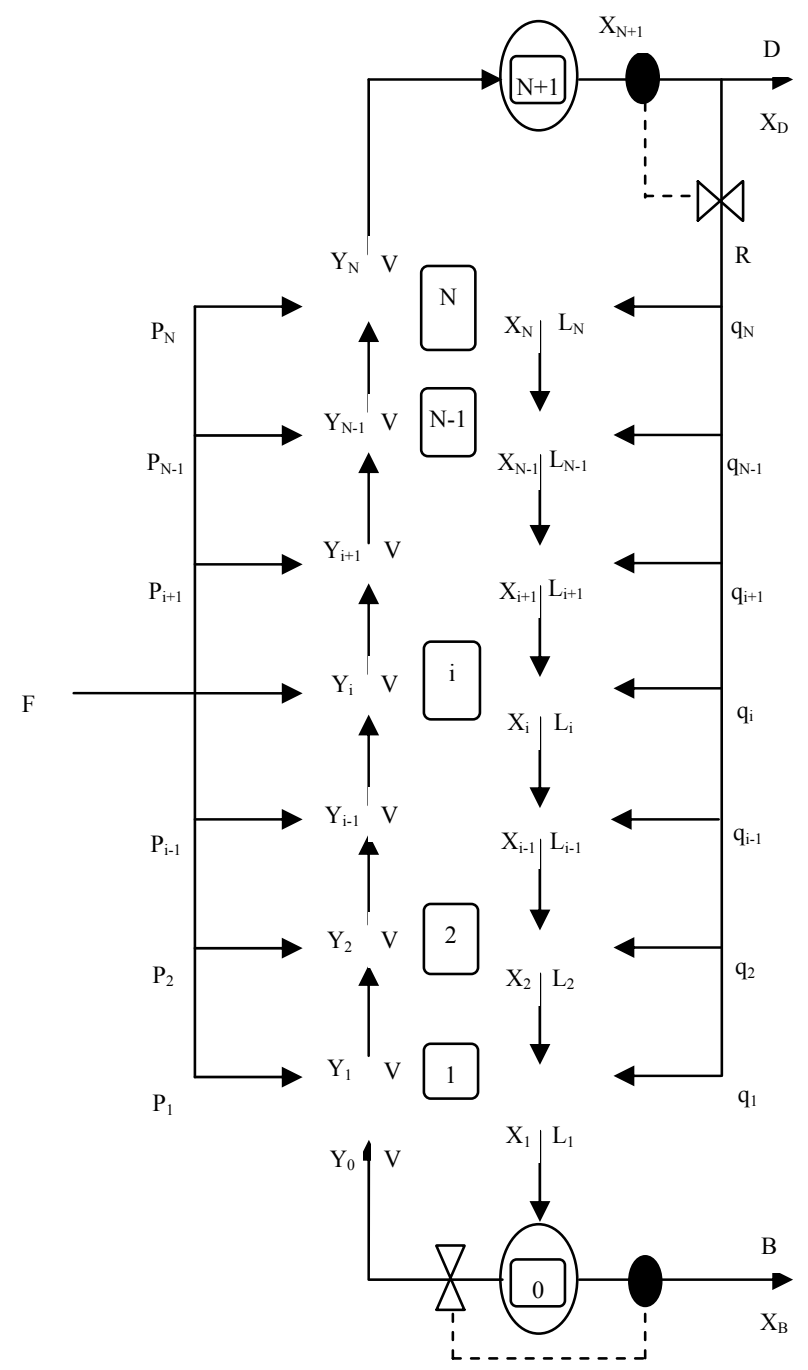

Fig. 1 Diagram of process (superstructure). 
feed location) of binary distillation column for the system described and intonation parameters associated controller control compositions and products bottom column distillate to be carried out effectively the separation of the components of the binary mixture. The proposed design must be optimal gain both economic criteria and the controllability of the process ensuring a feasible operation.

Being integrated, design optimization problem, the objectives are translated into:

- Minimize the total cost, which is the aim of designing processes and simultaneously;

- Minimize the ISE (integral square error), which is the goal of dynamic controllability.

To resolve this multiobjective problem becomes one of the objectives restriction $\left(o b j_{j} \leq \varepsilon\right)$, in this case the integral square error becomes part of the nonlinear constraints of the problem, which involves solving the problem MINLP-DAE (mixed integer no linear programming-differencial algebraic equations) to different values ranging from optimal controllable to the economic optimum, at this point the multiobjective problem becomes a problem of nonlinear programming with algebraic differential equations.

According to Schweiger, C. et al. [5], the following terms and restrictions to the process established first objective function of total cost (cost, expressed in \$) is related to design costs are assumed and utility through the following mathematical expression, where the cost or capital cost design $\left(\mathrm{C}_{\mathrm{c}}\right.$, expressed in $\left.\$\right)$ is related to the dimensions of the column and the cost of utility $\left(\mathrm{C}_{\mathrm{u}}\right.$, expressed in $\left.\$\right)$ with flows steam and reflux. Expression for the total cost:

$$
\begin{aligned}
& \cos t=7756 \cdot V_{S S}+3.075 \cdot\left(615+324 D_{C}^{2}+486 \cdot\left(6+0.76 N_{t}\right) D_{C}\right) \\
& +61.25 N_{t}\left(0.7+1.5 D_{C}^{2}\right)
\end{aligned}
$$

The mathematical expression for the purpose of controllability of the system is established based on the difference between the actual measured values of the compositions of distillate and bottom and their respective set points, where $\mu$ is the integral square error.

$$
\frac{d \mu}{d t}=t\left(x_{D}-x_{D}^{*}\right)^{2}+t\left(x_{B}-x_{B}^{*}\right)^{2}
$$

The initial condition for this problem is the steady state solution with control values at their nominal values. The dynamic state of the problem is induced by a step-like disturbance in the feed composition, expressed mathematically as follows:

$$
Z=0.45+\frac{0.9}{1+e^{10(t-10)}}
$$

The $\varepsilon$ restriction method is applied to multiobjective problem by imposing the following restriction on the endpoint:

$$
\mu\left(t_{f}\right) \leq \varepsilon
$$

\section{Results and Discussion}

\subsection{The Dynamic Behavior of ISE}

In Fig. 2, it is seen that the variation of controllability over the study period occurs in the range going from 0.0001 to 13.555 , which represent the values where the controllability has an appreciable impact on costs at the time of the integrated design.

\subsection{Relationship between Cost and Controllability of the System}

In Fig. 3, the curve indicates that the total cost is maintained without many variations from the upper assessed value $\varepsilon=13.555$, but begins to rise considerably when $\varepsilon=5.9808$, being the optimum value $\varepsilon=5.9473$. This way you get the value that provides a balance in both economic objectives and control objectives is $\varepsilon=5.9473$. It is obvious that a lower integral square error total design cost increased, because exercise better control over the system involves increased utility costs logically which affect the total cost.

\subsection{The Dynamic Behavior of the Controlled Variables, Distillate and Bottom Compositions}

Fig. 4 shows along the simulation period of 


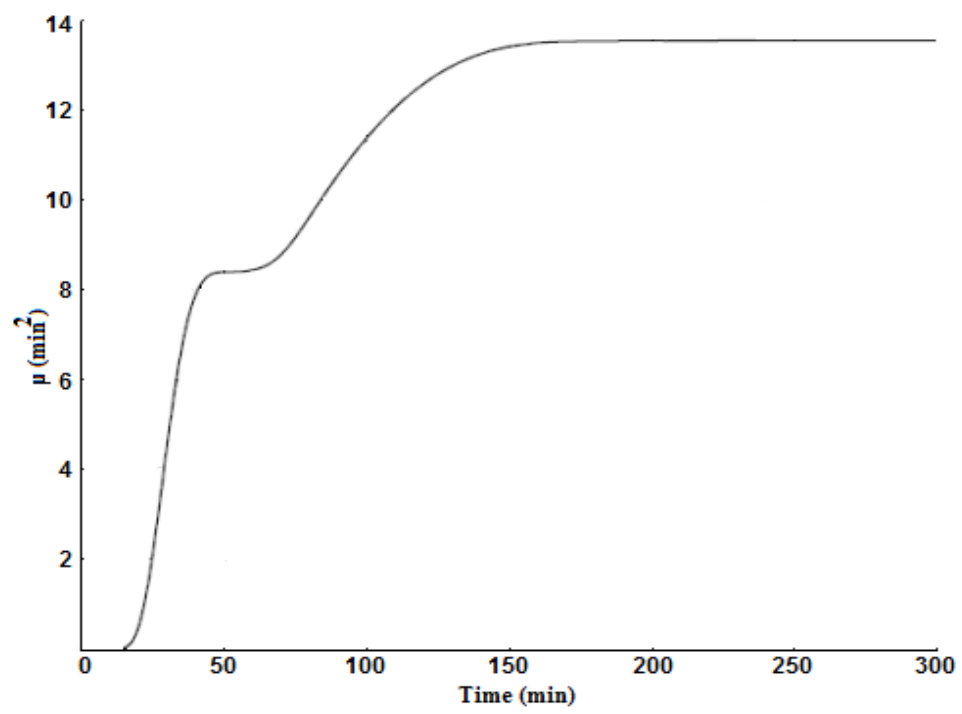

Fig. 2 Dynamic response ISE.

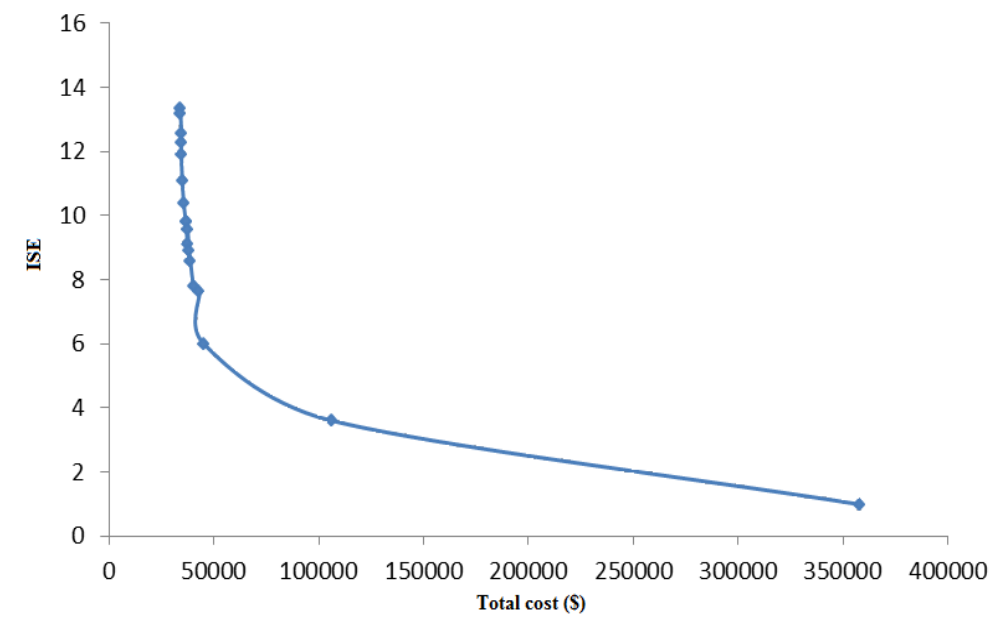

Fig. 3 Relationship between cost and controllability.

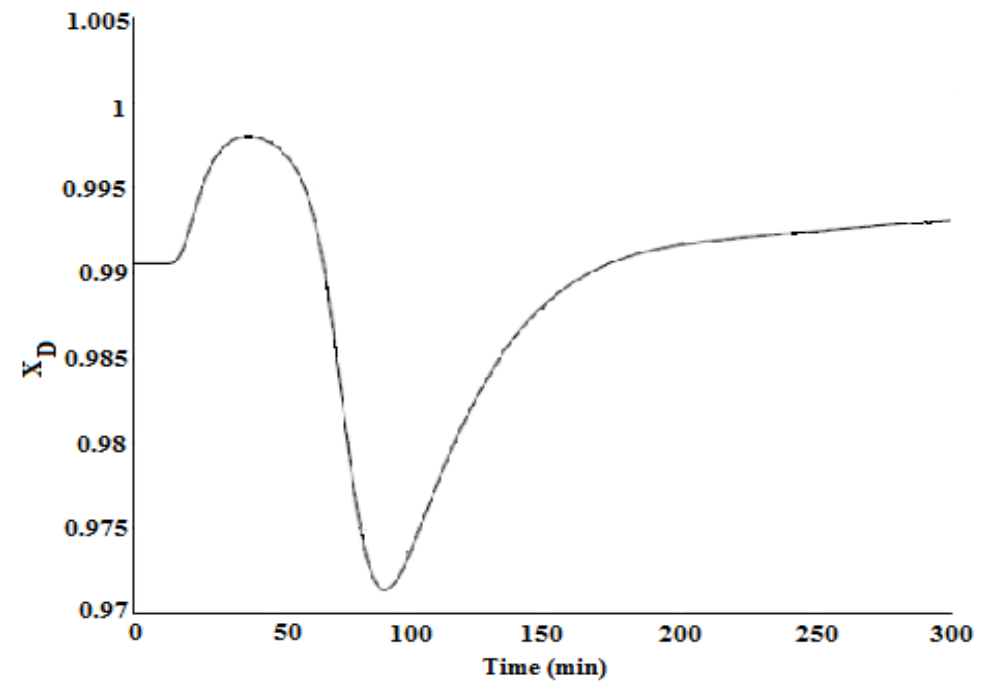

Fig. 4 Dynamic response of the composition of the distillate. 


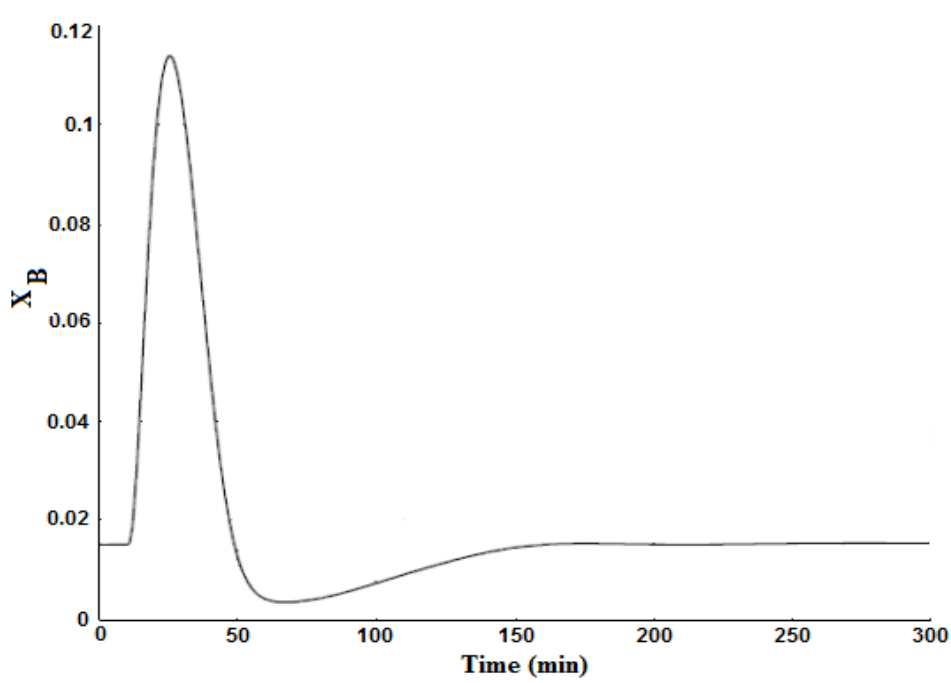

Fig. 5 Dynamic response of the composition of the bottom.

approximately $5 \mathrm{~h}$, as the composition stabilizes the system when the distillate is subjected to a disturbance both flow of the feed composition. The maximum value of deviation of the restriction distillate purity is found to reach an overshoot to a value of 0.97 . However, based on the total system response control indicates that after $1.8 \mathrm{~h}$ the composition rises to 0.98 and thereafter tends to stabilize at a higher mole fraction of 0.99 . Similarly occurs in the mole fraction curve residue or bottom shown in Fig. 5.

\section{Conclusions}

The systematic study of the application of different optimization techniques to integrated distillation column design, executed in this work provides two effective methodological strategies for the optimal solution of a distillation column which is a saturated liquid feed a binary mixture with a type partial reboiler and a total condenser.

For the most economically feasible methodological strategy the dynamic behavior of ISE presented a variation of controllability throughout the study period ranging from 0.77406 to 13.555 . The optimum controllability $\varepsilon$ (Ypsilon), which provides a balance in both economic objectives and control objectives is $\varepsilon=3.0624$.

Using the design methodology integrated system takes between $1.8 \mathrm{~h}$ and $2.5 \mathrm{~h}$ to stabilize. Higher number of defective products is obtained in the first case, while waiting for the system to reach steady state.

The integrated design studied system has better stability against perturbations.

The integrated design optimizes both initial investment costs, operating costs, system stability over time and control ranges and excellent quality products at the right time and in tune with environmental preservation.

\section{References}

[1] Skogestad, S. 1991. "Consistency of Steady-State Models Using Insight about Extensive Variables." Ind. Eng. Chem. Res. 30: 654-61.

[2] Skogestad, S., and Morari, M. 1987. "Control Configurations for Distillation Columns.” AIChE Journal 33: 1620-35.

[3] Gutiérrez, G., and De Prada, C. 2003. Integrated Design and Synthesis of Chemical Processes. Valladolid: University of Valladolid.

[4] Luyben, M. L., and Floudas, C. A. 1994. "Analyzing the Interaction of Design and Control." Comp. Chem. Eng. 18 (10): 933-69.

[5] Schweiger, C., and Floudas, C. 1997. "Interaction of Design and Control. Optimization with Dynamic Models." In Optimal Control Theory, Algorithms and Applications, edited by En Hager, W. W., and Pardalos, P. M. Kluwer Princeton: EEUU. 